\title{
OCORRÊNCIA DE INTERNAÇÕES HOSPITALARES POR DOENÇAS DO APARELHO CIRCULATÓRIO NO ESTADO DA BAHIA
}

\author{
Emmily Coelly Vieira*, Augusto Cesar Costa Cardoso**, Luciana Bilitário Macêdo***, Cristiane Maria \\ Carvalho Costa Dias**** \\ Autor Correspondente: Emmily Coelly Vieira - emmilyvieira11.1@bahiana.edu.br \\ * Acadêmica do Curso de Fisioterapia da Bahiana - Escola Bahiana de Medicina e Saúde Pública \\ ** Pós-doutorado em Saúde Coletiva pelo Instituto de Saúde Coletiva da Universidade Federal da Bahia \\ *** Mestre em Medicina e Saúde Humana pela Escola Bahiana de Medicina e Saúde Pública \\ **** Doutora em Medicina e Saúde Humana pela Escola Bahiana de Medicina e Saúde Pública
}

\section{Resumo}

Introdução: As Doenças do Aparelho Circulatório (DAC) em 2009 estavam entre as responsáveis por $28,7 \%$ dos óbitos em países em desenvolvimento e $26,6 \%$ nos países desenvolvidos. O custo direto relacionado ao manejo das DAC no Brasil é elevado, com impacto significativo no orçamento dos órgãos financiadores da saúde, especialmente quanto ao gasto com medicamentos, internações e na atenção secundária e terciária. Objetivo: Descrever as características sociodemográficas e o maior índice de internações hospitalares por doenças do aparelho circulatório no estado da Bahia. Materiais e métodos: Natureza quantitativa, do tipo descritiva, fundamentada em dados secundários, baseada em informações de saúde com dados coletados no Departamento de Informática do Sistema Único de Saúde (DATASUS), no período entre 2008 à 2013. A população alvo é de indivíduos com doenças do aparelho circulatório, com morbidade hospitalar, sendo incluídos no estudo pessoas de ambos os sexo, cor/raça, idade entre 40 e 79 anos, dos quais foram coletadas ocorrências envolvendo o aparelho circulatório. Resultados: Os maiores números de ocorrência da internação hospitalar geral por DAC no Sistema Único de Saúde na envolveram as cidades de Salvador e Feira de Santana, com destaque para a insuficiência cardíaca e hipertensão essencial na população entre 70 a 79 anos. Conclusão: A maior ocorrência de DAC foi insuficiência cardíaca na faixa etária de 70 a 79 anos, o sexo masculino teve maior morbidade hospitalar e, em relação à cor/raça observou-se homogeneidade na sua distribuição.

Palavras-chave: Doenças do aparelho circulatório; Ocorrência de internações hospitalares; Sistema Único de Saúde. 


\title{
HOSPITAL ADMISSIONS DUE TO CIRCULATORY SYSTEM DISEASES IN BAHIA
}

\begin{abstract}
Introduction: Circulatory System Diseases (CSD) in 2009 were among those responsible for $28.7 \%$ of deaths in developing countries and $26.6 \%$ in developed countries. The direct costs related to the management of CSD in Brazil is high, with significant impact on the budget of the health financing agencies, especially regarding the expenditure on drugs, hospitalizations and secondary and tertiary care. Objective: To describe the sociodemographic characteristics and the highest rate of hospitalizations for cardiovascular diseases in the state of Bahia. Materials and Methods: This research is quantitative, descriptive, based on secondary data, health information based on data collected in the Department of the Unified Health System (DATASUS), in the period between 2008 and 2013. The target population are individuals with cardiovascular diseases with hospital morbidity, being included in the study: gender (male and female), race (white, black, brown, yellow, indigenous), age (40 to 79 years old). Results: The highest rate of hospitalization due to CSD were in Salvador and Feira de Santana, with heart failure and essential hypertension in the population from 70 to 79 years old. Conclusion: The most prevalent CSD was the heart failure in 70 to 79 years old individuals. Males presented higher morbidity and among the races displayed homogeneous distribution.
\end{abstract}

Keywords: Circulatory diseases; Occurrence of hospital admissions; Unified Health System.

\section{INTRODUÇÃO}

As Doenças do Aparelho Circulatório (DAC) são um fenômeno global e desde o século XIX e XX, atingem países desenvolvidos e em desenvolvimento. As DAC surgiram como resultado da urbanização e industrialização, destacando-se como a principal causa de morte. ${ }^{(1,2)}$ De acordo com a Organização Mundial da Saúde, as DAC no ano de 2009 estavam entre as responsáveis por $28,7 \%$ dos óbitos nos países em desenvolvimento e $26,6 \%$ nos países desenvolvidos. ${ }^{(3)}$

O custo direto relacionado ao manejo das DAC no Brasil é elevado, com impacto significativo no orçamento dos órgãos financiadores da saúde, especialmente quanto ao gasto com medicamentos, internações e na atenção da alta complexidade.(4) $O$ valor dos serviços hospitalares no Nordeste entre Janeiro de 2008 e Julho de 2015 foi equivalente a 2.460.057.417,04 e no Estado da Bahia $579.984 .470,55$ (DATASUS). ${ }^{(5)}$

Dentre as principais DAC se destacam as doenças cerebrovasculares, hipertensão arterial elevada, doença arterial periférica, cardiopatia reumática, cardiopatia congênita e insuficiência cardíaca; sendo que os fatores de riscos para essas doenças são o tabaco, inatividade física, dieta inadequada, uso abusivo do álcool, ${ }^{(6)}$ além dos fatores sociais como baixa escolaridade, classe social e renda familiar ou dos biológicos, como idade, sexo, cor da pele/raça e história familiar de DAC. ${ }^{(1)}$

A doença cerebrovascular que mais incapacita a população brasileira é o Acidente Vascular Cerebral (AVC), com índices de 70\% para incapacidade laboral e 50\% para realizações das atividades de vida diária. (7) Atinge, em sua maioria, a população acima de 
40 anos, sendo a população com idade a partir dos 65 anos a que mais sofre sequelas, hospitalizações e óbitos. ${ }^{(8)}$ A Hipertensão Arterial Sistêmica (HAS) é considerada a mais frequente das doenças cardiovasculares, principal fator de risco para desencadeamento do acidente vascular encefálico e do infarto agudo do miocárdio. (9) A HAS caracteriza-se como uma doença multifatorial e multicausal, com níveis pressóricos elevados acima dos níveis de normalidade, sendo predominante na fase adulta. ${ }^{(10)} \mathrm{De}$ acordo com o ministério da saúde, a hipertensão arterial ocupa o primeiro lugar para aposentadoria por invalidez, com índice de 20,4\%.(11)

Em relação às doenças vasculares, a Doença Arterial Periférica (DAP), é ocasionada pelo acúmulo de gordura na parede das artérias periféricas, a claudicação intermitente é o seu principal sintoma, caracterizada por dor e desconforto ao caminhar, ${ }^{(12)}$ acomete a aorta e seus ramos, possui altas taxas de morbidade e mortalidade, com prevalência de $10 \%$ a $25 \%$ em pessoas com mais de 55 anos e $80 \%$ para a faixa etária acima de 70 anos. ${ }^{(13)}$

A Febre Reumática (FR) é proveniente do germe estreptococos e pode ocasionar secundariamente a cardiopatia reumática, ${ }^{(14)}$ afeta a população pobre, que habita em lugares úmidos e condições de vida precária, ainda no início da idade escolar. ${ }^{(15)}$ Segundo o DATASUS, no Brasil entre Janeiro e Junho de 2015 foram gastos com serviços hospitalares para febre reumática um total de 1.021.795,95 de reais, as regiões que mais gastam com serviços hospitalares são a região Sudeste com somatório de 388.497,51, seguido da região Nordeste com $346.660,75 .{ }^{(5)} \mathrm{A}$ Insuficiência Cardíaca (IC) é um problema que ocorre quando, por qualquer doença, o coração deixa de bombear o sangue na quantidade necessária à manutenção do corpo. ${ }^{(16)}$ No Brasil são 1.897.447 internações entre Janeiro e Junho de 2015 (DATASUS). ${ }^{(5)}$

Diante dessa problemática de saúde, o objetivo desse estudo é descrever as características sociodemográficas e o maior índice de internações hospitalares por doenças do aparelho circulatório no estado da Bahia entre 2008 e 2013 , apontar as doenças cardiovasculares que mais afetam a população e, através dos resultados obtidos, ampliar o conhecimento sobre o tema, a fim de possibilitar elaborar estratégias de prevenção para as morbidades hospitalares, baseada em estudos de dados públicos.

\section{METODOLOGIA}

A presente pesquisa é de natureza quantitativa, do tipo descritivo, fundamentada em dados secundários no período entre 2008 e 2013 , baseada em informações de saúde, com dados coletados no Departamento de Informática do Sistema Único de Saúde (DATASUS), a partir do Sistema de Informações Hospitalares (SIH/SUS). Apresenta como instrumento, o formulário de Autorização de Internação Hospitalar (AIH). A área de estudo foi o estado da Bahia, que possui uma população de 14.175.341 habitantes, localizada na região Nordeste do Brasil, o estudo apresenta como população alvo os indivíduos com doenças do aparelho circulatório.

A seleção da amostra foi realizada a partir da plataforma Informações de Saúde (TABNET) e selecionados indicadores epidemiológicos e morbidade hospitalar, com a opção: Geral, por local de residência - a partir de 2008. As variáveis desse estudo foram: sexo (masculino e feminino), raça (brancos, pretos, pardos, amarelos, indígenas), idade (40 à 79 anos). Foram excluídas as morbidades do CID 10 que não se enquadrem como doença do aparelho circulatório no estado da Bahia.

\section{RESULTADOS}

A ocorrência de internação hospitalar geral por DAC no SUS entre homens e mulheres, período entre 2008 e 2013 , está registrada no gráfico 1, mostrando um destaque para a insuficiência cardíaca com 82.191 casos, na população entre 70 a 79 anos, seguida de hipertensão essencial com 46.273 casos. A hipertensão essencial foi mais prevalente em 
indivíduos com idade entre 40 a 49 anos, o acidente vascular não especificado em hemorrágico ou isquêmico, apresentou 39.286 casos, afetando indivíduos entre 70 a 79 anos, o infarto agudo do mio- cárdio apresentou 20.726 casos, doenças isquêmicas do coração com 19.015 casos, ambos atingindo a maior parte da população com idade entre 50 a 59 anos.

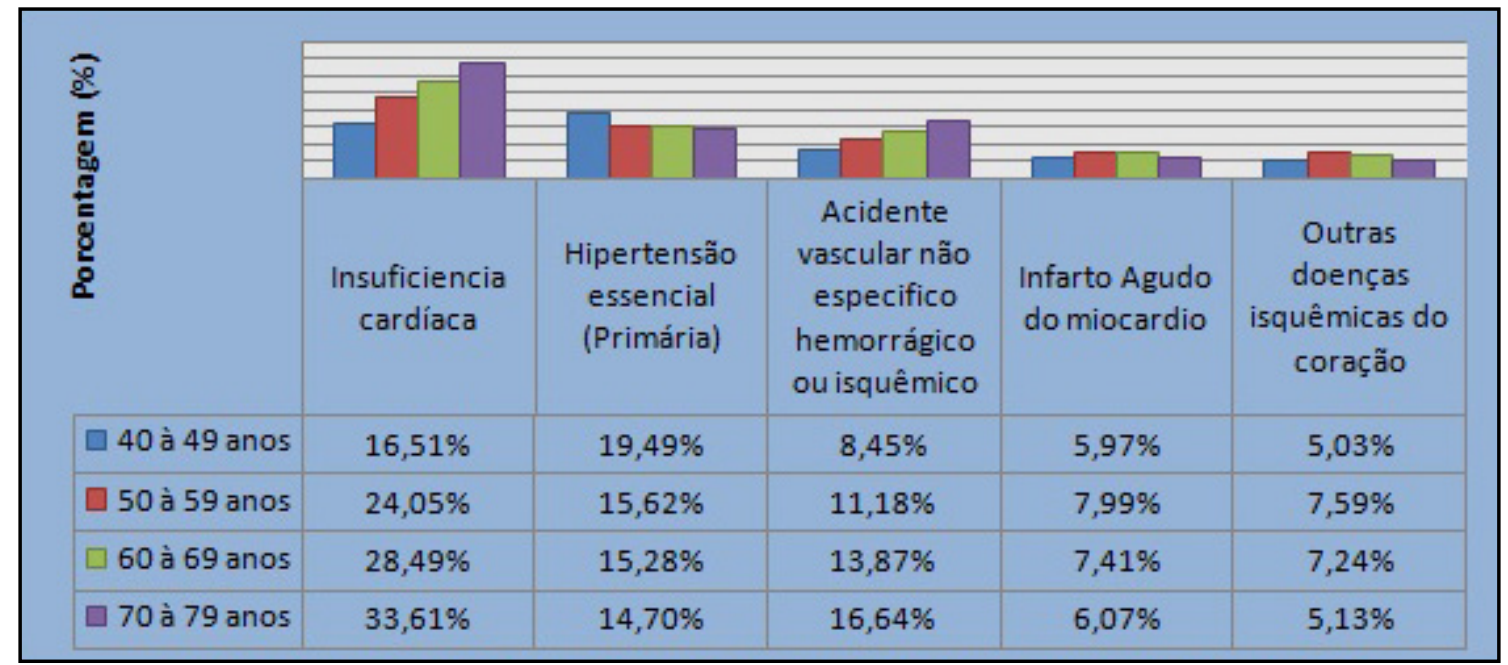

Gráfico 1 - Proporção de internação hospitalar geral por DAC, segundo CID-10 e faixa etária, Estado da Bahia 2008-2013 Fonte: Ministério da Saúde - Sistema de Informações Hospitalares do SUS.

No gráfico 2, a Insuficiência cardíaca apresenta a maior causa de internação entre os homens com 44.146 casos, mais frequente na faixa etária de 60 a 69 anos, hipertensão essencial primária obtendo 16.716 casos, essa mais frequente em indivíduos com 40 à 49 anos. $O$ tipo do acidente vascular cerebral não especificado hemorrágico ou isquêmico ocorreu em 19.844 casos, maioria em homens com idade entre 70 e 79 anos, o infarto agudo do miocárdio com 12.303 casos, ocorreu em maior parte em indivíduos entre 50 a 59 anos, e outras doenças isquêmicas do coração 10.767 casos, entre 50 a 59 anos.

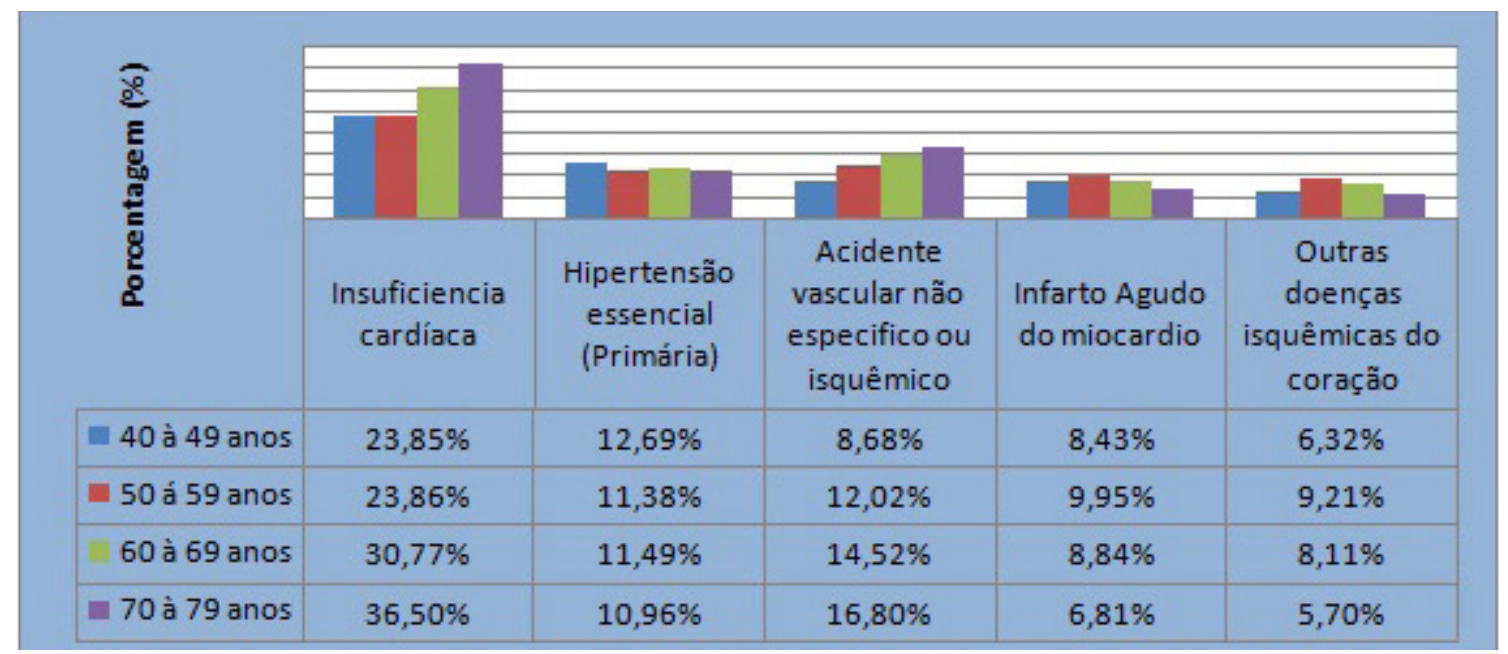

Gráfico 2 - Proporção de internação hospitalar por sexo masculino, segundo a Classificação Internacional de Doenças (CID-10) e faixa etária, no estado da Bahia, 2008- 2013. 
O gráfico 3, mostra que a primeira causa de internação hospitalar na população feminina é a IC com faixa etária entre 70 e 79 anos, seguida da hipertensão arterial primária, mais ocorrente na ida- de entre 40 e 49 anos, do acidente vascular não especifico hemorrágico ou isquêmico entre 60 e 69 anos, veias varicosas das extremidades inferiores entre 50 e 59 anos e infarto agudo do miocárdio entre 40 e 49 anos.

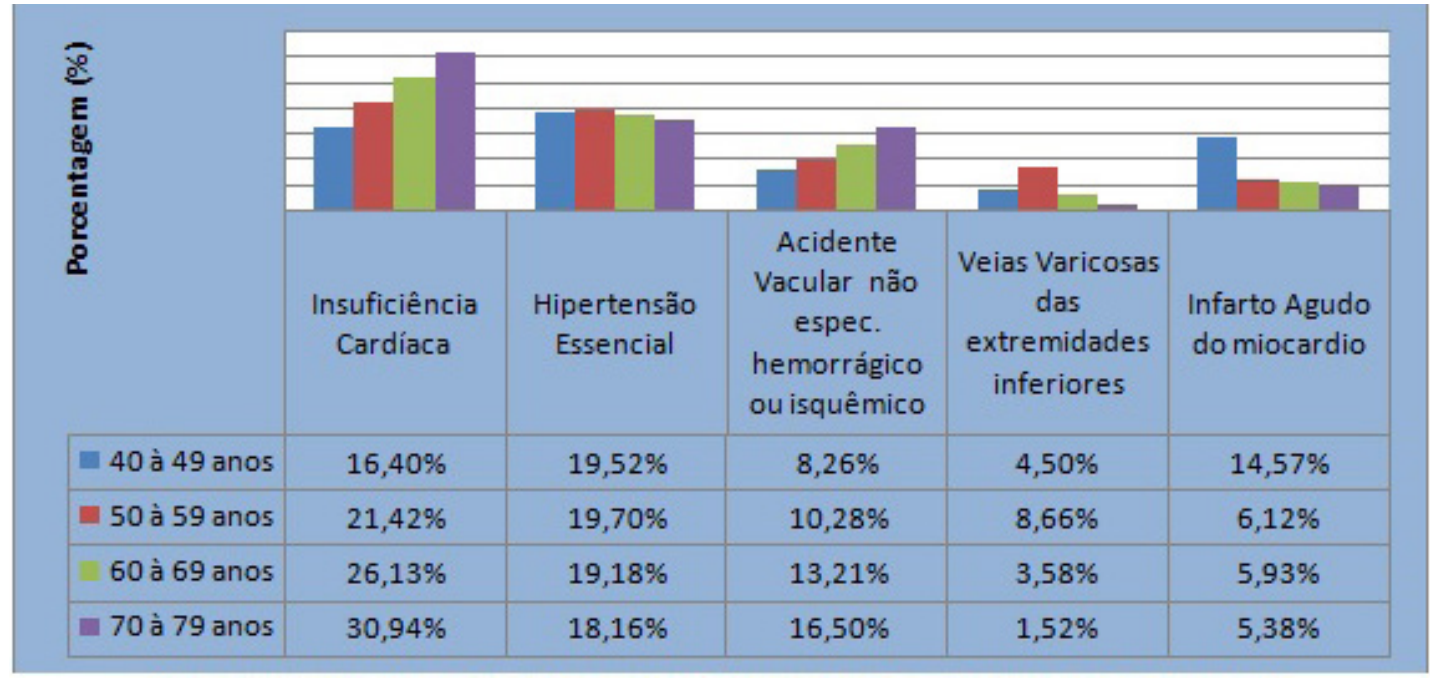

Gráfico 3 - Proporção de morbidade hospitalar por raça, entre o sexo feminino, segundo CID-10, faixa etária no estado da Bahia, 2008- 2013

Fonte: Ministério da Saúde - Sistema de Informações Hospitalares do SUS.

$\mathrm{Na}$ tabela 1, os dados mostram que a internação por IC ocorre em maior parte em mulheres pardas (29,09\%), a Hipertensão Essencial Primária (HEP) atinge em maior parte as mulheres pretas $(29,23 \%)$ e Veias Varicosas (VV) das extremidades inferiores (7,29\%). O AVC não especificado hemorrágico mulheres amarelas $(16,38)$, não houve da- dos das mulheres indígenas. O Infarto Agudo do Miocárdio (IAM) em mulheres brancas (7,88\%) e outras doenças cardíacas em mulheres indígenas $(6,91 \%)$. Não houve notificação da raça indígena para o diagnóstico de varizes varicosas e infarto agudo do miocárdio.

Tabela 1 - Proporção de internação hospitalar por DAC, relacionado à raça, sexo feminino, segundo a Classificação Internacional de Doenças (CID-10) faixa etária e raça, no estado da Bahia, 2008-2013

\begin{tabular}{cccccc}
\hline \multicolumn{7}{c}{ RAÇA } \\
\hline BRANCA & PRETA & PARDA & AMARELA & INDÍGENA \\
\hline ICC & $27,96 \%$ & $25,87 \%$ & $29,09 \%$ & $27,68 \%$ & $20,83 \%$ \\
HEP & $20,96 \%$ & $29,23 \%$ & $24,82 \%$ & $27,12 \%$ & $41,67 \%$ \\
AVC & $12,15 \%$ & $10,97 \%$ & $11,41 \%$ & $16,38 \%$ & $4,17 \%$ \\
VV & $5,76 \%$ & $7,29 \%$ & $4,58 \%$ & $2,26 \%$ & $-\cdots-----$ \\
IAM & $7,88 \%$ & $5,38 \%$ & $6,21 \%$ & $4,07 \%$ & $-\cdots----$ \\
DC & $6,91 \%$ & $3,44 \%$ & $5,13 \%$ & $2,91 \%$ & $9,09 \%$ \\
\hline
\end{tabular}

Fonte: Ministério da Saúde - Sistema de Informações Hospitalares do SUS. 
Na tabela 2, é possível notar que a insuficiência cardíaca é mais ocorrente em homens amarelos (44,19\%), a hipertensão essencial primária afeta os homens indígenas $(27,27 \%)$, o AVC não especifico hemorrágico ou isquêmico hospitaliza em maior parte os homens brancos $(15,70 \%)$, assim como o infarto agudo do miocárdio (7,88\%), e outras doenças isquêmicas do coração revela uma maior frequência os homens indígenas (9,09\%).

Tabela 2 - Proporção de internação hospitalar decorrente de DAC por raça, entre o sexo masculino, segundo a Classificação Internacional de Doenças (CID-10) faixa etária e raça, no estado da Bahia, 2008-2013

\begin{tabular}{cccccc}
\hline \multicolumn{7}{c}{ RAÇA } \\
\hline & BRANCA & PRETA & PARDA & AMARELA & INDÍGENA \\
\hline ICC & $32,91 \%$ & $40,63 \%$ & $36,75 \%$ & $44,19 \%$ & $31,82 \%$ \\
HEP & $12,96 \%$ & $13,71 \%$ & $15,76 \%$ & $15,12 \%$ & $27,27 \%$ \\
AVC & $12,94 \%$ & $12,88 \%$ & $13,17 \%$ & $15,70 \%$ & $9,09 \%$ \\
IAM & $7,88 \%$ & $5,38 \%$ & $6,21 \%$ & $4,07 \%$ & - \\
DC & $6,91 \%$ & $3,44 \%$ & $5,13 \%$ & $2,91 \%$ & $9,09 \%$ \\
\hline
\end{tabular}

Fonte: Ministério da Saúde - Sistema de Informações Hospitalares do SUS.

$I C C=$ Insuficiência Cardíaca; HEP= Hipertensão Essencial Primária; $V V=$ Veias Varicosas; AVC=

Acidente Vascular Cerebral; IAM= Infarto Agudo do Miocárdio; DC = Outras doenças cardíacas isquêmicas.

O gráfico 4 apresenta dados a partir dos números de hospitalizações por DAC de acordo com região de saúde/município. Foi observado que a cidade de Salvador obteve o maior número de hospitalizações pelo SUS (19\%), Feira de Santana em segundo lugar (7\%), Jequié e Vitória da Conquista em terceiro lugar (6\%); Jacobina, Itabuna, Santo Antônio de Jesus em quarto lugar (5\%); Irecê,
Ilhéus, Serrinha em quinto lugar (4\%), Teixeira de Freitas, Guanambi, Juazeiro, Brumado, Itapetinga, Alagoinhas, Senhor do Bonfim, Seabra em sexto lugar (3\%); Valença, Porto Seguro, Ribeira do Pombal, Itaberaba, Santa Maria da Vitória em sétimo lugar (2\%), a menor ocorrência Paulo Afonso em oitavo lugar (1\%).
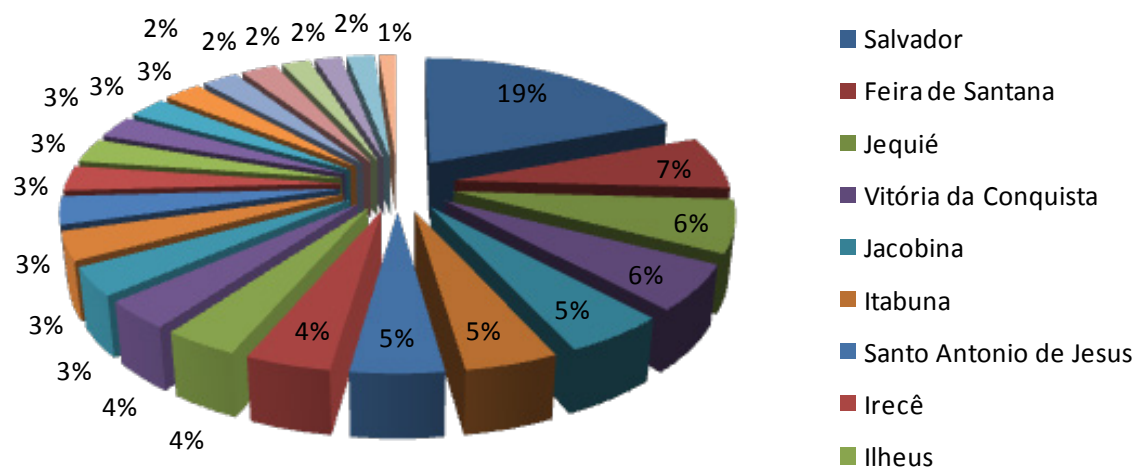

Gráfico 4 - Proporção de internação hospitalar do SUS decorrente de DAC de acordo com Região de saúde / Município no estado da Bahia, 2008 a 2013

Fonte: Ministério da Saúde - Sistema de Informações Hospitalares do SUS (SIH/SUS) 


\section{DISCUSSÃO}

O presente estudo revela que a morbidade hospitalar mais frequente por DAC no estado da Bahia, período de 2008 a 2013, foi a insuficiência cardíaca, na população mais idosa, seguida da hipertensão essencial primária em indivíduos mais jovens. A maior ocorrência das internações hospitalares ainda afetam mais os homens, apesar de que as mulheres vem apresentando um aumento das internações por DAC nesse estado.

Esses dados são confirmados pela Secretária de Saúde do Estado da Bahia, que aponta que, nos últimos três anos, o sexo feminino desponta com maiores registros de internações por doenças cardiocirculatórias em relação ao sexo masculino. Este achado pode ser justificado pela mudança do estilo de vida dessa população, representada pela dupla jornada de trabalho, aumento de tarefas diárias, resultando em aumento do estresse, tabagismo, má alimentação e sedentarismo, ${ }^{(17)}$ fatores de risco para as doenças cardiovasculares. Um estudo realizado a partir do triênio 1998-2000 mostrou que as hospitalizações por insuficiência cardíaca no Brasil foram maiores no sexo feminino, com idade entre 70 e 79 anos, ${ }^{(18)}$ sendo possível observar uma equiparidade com os resultados dessa pesquisa em relação à faixa etária.

Uma pesquisa realizada a partir da análise da mortalidade e das internações por doenças cardiovasculares em Niterói, entre 1998 e 2007, mostrou que o coeficiente de internação de internação por IC foi $2.593,44$, entre 1998 e 2000 para a faixa etária maior que 80 anos, diminuindo significantemente os índices para 321,15 , entre 2005 e 2007 , com coeficiente maior para o sexo masculino. ${ }^{(19)}$ Godoy, Silveira, Segalla e Almeida evidenciaram no município de São Paulo registrado pelo SUS, entre 1992 e 2010, que o sexo feminino destacou-se na idade entre 60 a 79 anos, porém foi observada uma redução da ocorrência de morbidade hospitalar com significância estatística, quando comparado o período de 1992 com 2010. ${ }^{(20)}$ Há disparidade da frequência da morbidade hospitalar por ICC em relação ao sexo, entre as regiões brasileiras.
O AVC gera incapacidade laboral e grande déficit econômico, responsável por 68 mil mortes por ano. ${ }^{(21)}$ Nesse estudo foi possível verificar que o AVC não especificado entre hemorrágico ou isquêmico, afeta indivíduos do sexo masculino com idade entre 70 e 79 anos, e o sexo feminino entre 60 e 69 anos, ocupando quarto lugar de destaque entre ambos, corroborando com os resultados de um estudo realizado com residentes do município do Paraná. ${ }^{(22)}$

A frequência de internamento hospitalar para hipertensão essencial primária é maior na população feminina, faixa etária de 50 a 59 anos (19,70\%) em relação ao sexo masculino (11,38\%), essa prevalência é demonstrada por Santos et al. em uma pesquisa de internações por hipertensão arterial no Brasil $^{(23)}$ e VI Diretrizes Brasileira de Hipertensão. (24) De acordo com os dados coletados, o infarto agudo do miocárdio ocupa o quarto lugar em internações hospitalares, apresentando maior ocorrência entre o sexo masculino, semelhante a esse resultado, foi apontado o predomínio para o sexo masculino. ${ }^{(25,26)} \mathrm{A}$ despeito desse cenário, é relevante observar a frequência crescente dessa patologia no sexo feminino.

As veias varicosas de extremidades inferiores ocuparam o quarto lugar por internações, em muIheres com maior índice na faixa etária de 50 a 59 anos. Comumente, estima que $20 \%$ à $25 \%$ das muIheres são afetadas, ${ }^{(27)}$ no entanto, não houve resultados na literatura para afirmar a ocorrência de internações quanto ao sexo e faixa etária. A maior prevalência de doenças do aparelho circulatório de acordo com a raça apresentou resultados homogêneos, porém, ainda faltam estudos que evidenciem a ocorrência de internação por DAC entre as raças.

Os municípios de Salvador e Feira de Santana apresentaram posição de destaque no estado da Bahia. De acordo com o IBGE, ${ }^{(28)}$ estima-se que Salvador apresenta uma população de 2.675 .656 habitantes, Feira de Santana apresenta 556.642 habitantes, Jequié 151.895 habitantes, Vitória da Conquista 306.866 habitantes. Diante dos números apresentados é possível perceber que a capital da 
Bahia apresenta aproximadamente uma população cinco vezes maior que o município de Feira de Santana, no entanto é somente 2,5 vezes maior a ocorrência de internações por DAC, já os municípios que apresentaram terceiro e quarto lugar em internações por DAC, apresentam inversão de dados em relação ao número populacional, ou seja, Jequié apesar de índice populacional menor, seus habitantes são mais atingidos que os habitantes de Vitória da Conquista.

O presente estudo apresenta limitações devido à utilização de dados secundários no SIH/SUS, o que pode apresentar subnotificações e pelo fato de poder apresentar informações distorcidas, já que nem todos os municípios do estado podem estar informando ao banco de dados informações precisas, devido aos conflitos de interesse que envolvem a gestão da saúde pública e privada sobre a visão financeira de saúde e não realmente epidemiológica, além de ser importante ressaltar que alguns municípios podem apresentar condições precárias de tecnologia.

\section{CONCLUSÃO}

De acordo com o presente estudo, maior ocorrência de morbidade hospitalar por DAC são ocasionados primeiramente pela insuficiência cardíaca para ambos os sexos, com homogeneidade de ocorrência por cor/raça. As cidades com maiores índices de internamento por DAC são Salvador e Feira de Santana.

\section{REFERÊNCIAS}

1. Gersh BJ, Sliwa K, Mayosi BM, Yusuf S. Novel therapeutic concepts: the epidemic of cardiovascular disease in the developing world: global implications. Eur Heart J. 2010;31(6): 642-8.

2. Colombo RCR, Aguillar OM. Estilo de vida e fatores de risco de pacientes com primeiro episódio de infarto agudo do miocárdio. Rev Lat Am Enfermagem. 1997;5(2):69-82.

3. World Health Organization. Global status report on no communicable diseases. Geneva: WHO; 2O1O. Available from: http://www.who. int/nmh/publicatinos/ncd_report_en.pdf

4. Andrade JP, Mattos LAP, Carvalho AC, Machado CA, Oliveira GMM. Programa Nacional de Qualificação de Médicos na Prevenção e Atenção Integral às Doenças Cardiovasculares. Arq Bras Cardiol. 2013;100(3):203-211

5. Ministério da Saúde (BR). Secretaria Executiva DATASUS [acesso em ago. 2Ol5]. Informações de Saúde. Informações epidemiológicas e morbidade. Disponível em: www.datasus.gov.br

6. World Health Organization. Noncommunicable Diseases and Mental Health. Integrated management of cardiovascular risk: report of a WHO meeting; 2002 July 9-12; Geneva: World Health Organization; 2002.

7. Sociedade Brasileira de Doenças Cerebrovasculares. Acidente Vascular Cerebral. 2O12. Disponível em: http://www.sbdcv.org.br/ publica_AVC

8. Furukawa TS, Mathias TA, Marcon SS. Mortalidade por doenças cérebro vasculares por residência e local de ocorrência do óbito: Paraná, Brasil, 2007. Cad. Saúde Pública. 2011;27(2);327-334.

9. Ministério da Saúde (BR). Hipertensão Arterial Sistêmica [Internet]. Brasília (DF): Ministério da Saúde; 2006. (Caderno de Atenção Básica; 15). Disponível em: http://bvsms.saude.gov.br/bvs/ publicacoes/caderno_atencao_basical5.pdf

10. Kohlmann Jr O, Costa GA, Carvalho MHC, Chaves Jr HC, Machado CA, Praxedes JN et al. III Consenso Brasileiro de Hipertensão Arterial. Arq Bras Endocrinol Metab [Internet]. 1999;43(4):257-286.

11. Ministério da Saúde (BR); Organização Pan-Americana da Saúde/Brasil. Doenças relacionadas com o trabalho: diagnóstico e condutas: manual de procedimentos para os serviços de saúde [Internet]. Brasília (DF): Ministério da Saúde; 2001. p. 277-306. (Série A, Normas e manuais técnicos; 114). Disponível 
em: http://bvsms.saude.gov.br/bvs/publicacoes/ doencas_relacionadas_trabalhol.pdf

12. Schann D'Argold B, Mandeli NCB. Conduta na doença arterial periférica em pacientes diabéticos. Revista da Sociedade Brasileira de Cardiologia do Rio Grande do Sul. 2004;8(2).

13. Sociedade Brasileira de Angiologia e de Cirurgia Vascular Regional São Paulo. Doença arterial obstrutiva periférica. Disponível em: http://sbacvsp.com.br/ index.php/homepage/ doencasvasculares/164-doenca-arterialobstrutivaperierica.html

14. Sociedade Brasileira de Cardiologia. Diretrizes brasileiras para o diagnóstico, tratamento e prevenção da febre reumática. Arq Bras Cardiol. 2009; 93(3 supl.4):1-18.

15. Chaves A. Cardiopatia reumática. Porto Alegre: Departamento Estadual de Saúde do Estado do Rio Grande do Sul; 2007 [Acesso em 2015 ago]. Disponível em: http://www.cardiol.br/tunel/ ago/new/OO7.pdf

16. Instituto Brasileiro de Geografia e Estatística [Internet]. Um panorama da saúde no Brasil, acesso e utilização dos serviços, condições e fatores de risco, proteção à saúde 2008. Rio de Janeiro: IBGE; 2010 [acesso em 2015 jul]. Disponível em: http:www.ibge.gov.br

17. Brandão C. SBC-BA esclarece a população feminina sobre os riscos das doenças cardiovasculares [Internet]. [acesso em 2016 jan]. Disponível em: http://socieda de.cardiol.br/ $\mathrm{ba/noticias/doencascardiovasculares.asp}$

18. Lessa I. Epidemiologia da hipertensão arterial sistêmica e da insuficiência cardíaca no Brasil. Rev Bras Hipertens.2O01;8(4):383-392.

19. Rosa MLG, Giro C, Alves TO, Moura EC, Lacerda LS, Santanna LP, et al. Análise da mortalidade e das internações por doenças cardiovasculares em Niterói, entre 1998 e 2007. Arq Bras Cardiol. 2011;96(6):477-483.

2O. Godoy HL, Silveira JÁ, Segalla E, Almeida DR. Hospitalização e mortalidade por insuficiência cardíaca em hospitais públicos no município de São Paulo. Arq Bras Cardiol. 2011;97(5):4O2407.

21. Ministério da Saúde (BR). Acidente vascular cerebral. 2012. Disponível em: http://www.brasil. gov.br/saude/2O12/O4/acidente-vascularcerebral-avc

22. Pilger C, Lentski MH, Vargas G, Baratiere T. Causas de internação hospitalar de idosos residentes em um município do Pará, uma análise dos últimos 5 anos. Rev Enferm UFSM. 2011;1(3):394-4O2.

23. Santos SS, Vasconcelos DFSA. Hospitalizações por hipertensão arterial essencial em caráter de urgência no Brasil, 2008-2O12. Rev Ciênc Méd Biol. 2013;12(esp.):465-471.

24. Sociedade Brasileira de Cardiologia; Sociedade Brasileira de Hipertensão; Sociedade Brasileira de Nefrologia. VI Diretrizes Brasileiras de Hipertensão. Arq Bras Cardiol. 2010;95(1 Supl 1):I-III.

25. Santos ESS, Minuzzo L, Pereira MP, Castillo MTC, Palácio MAG, Ramos RF, et al. Registro de síndrome coronariana aguda em um centro de emergências em cardiologia. Arq Bras Cardiol. 2006; 87 (5):597-602

26. Piegas LS, Avezum V, Guimarães HP, Reis HJL, Santos ES, Knobel M, et al. Comportamento da síndrome coronariana aguda. Resultados de um registro brasileiro. Arq Bras Cardiol. 2013;10O(6):502-510.

27. Sociedade Brasileira de Angiologia e de Cirurgia Vascular. Varizes dos membros inferiores: tratamento cirúrgico. São Paulo: Associação Médica Brasileira; 2012.

28. Instituto Brasileiro de Geografia e Estatística. [Internet]. Sinopse do Censo Demográfico 2010 Brasil. Rio de Janeiro: IBGE; 2011 [acesso em 2016 mar]. Disponível em: http:// www.censo2O1O.ibge.gov.br/sinopse/index. php?dados $=6 \& u f=0 O$ 